\title{
Is pseudoacetabulum an important factor determining SSTO application in total hip arthroplasty for Crowe IV hips? a retrospective cohort study
}

\author{
Haiwen Peng ${ }^{1}$, Guogiang Zhang ${ }^{1}$, Chi Xu' ${ }^{1}$, Tianhao Wang ${ }^{1}$ and Yan Wang ${ }^{1,2^{*}}$
}

\section{Introduction}

In terms of developmental dysplasia of the hip (short for DDH), several classification systems have been proposed [1-5]. Most literature is published applicating Crowe's classification system, because it could be suggestive and instructive for surgery. In 1979, Crowe et al. [1] proposed a four-type system classifying the degree of hip dislocation regarding the ratio of proximal displacement distance of the femoral head in relation to the height of the pelvis or the femoral head, resulting in a calculated coefficient, which converts into one of the four types. Crowe practiced as a hand surgeon after that, so this system was used worldwide and never ameliorative for improvement. In 1987, Eftekhar et al [6] proposed a four-type classification system based on the presence or absence of pseudoacetabulum and overlap between the true acetabulum and the false one. In 1996, Hartofilakidis et al. [4] used the pathology of the dysplastic acetabulum to distinguish between three different types of dysplasia, with subtypes discriminating between a primary and a secondary acetabulum and the relation of the head in relation to these structures. In 2008, Hartofilakidis [7] proposed subtypes on the presence or absence of false acetabulum, and in 2013, he analyzed the clinical outcome of THA based on different subtypes [8]. With the classification of Crowe being increasingly popular, especially in the English literature, it may be the best choice for a classification system with the data available today [9]. In 2016, Ma et al. reported different results between hips of Crowe type IV subtypes on the presence or absence of false acetabulum [10].

\footnotetext{
* Correspondence: wangyansurgeon@sina.com

${ }^{1}$ Department of Orthopaedic Surgery, Medical School of Chinese People's Liberation Army, Army Medical University of Chinese People's Liberation Army, No. 28 Fuxing Road, Haidian District, Beijing 100853, China

${ }^{2}$ Department of Orthopaedic Surgery, Medical School of Chinese People's Liberation Army, General Hospital of Chinese People's Liberation Army, Beijing, China
}

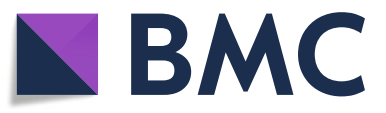

(c) The Author(s). 2019 Open Access This article is distributed under the terms of the Creative Commons Attribution 4.0 International License (http://creativecommons.org/licenses/by/4.0/), which permits unrestricted use, distribution, and reproduction in any medium, provided you give appropriate credit to the original author(s) and the source, provide a link to the Creative Commons license, and indicate if changes were made. The Creative Commons Public Domain Dedication waiver (http://creativecommons.org/publicdomain/zero/1.0/) applies to the data made available in this article, unless otherwise stated. of deve whe is very difficut to reconst which is very difficult to reconstruct the original acetabulum and reset the rotation center to equal both legs and avoid nerve injury after total hip arthroplasty (short for THA). Placing the acetabular component at the true hip center has been shown to provide successful long-term results [11]. However, placement of the acetabular component in the original acetabulum location may result in nerve injury by excessive leg lengthening. This can be addressed by subtrochanteric shortening osteotomy (short for SSTO) first described as a treatment for congenital dislocation of the hip in older children [12], which demands accurate anatomical knowledge and technical expertise for durable results. It was later applied to patients with THA [13]. The shortening subtrochanteric osteotomy (short for SSTO) combined with total hip arthroplasty was a good way to solve the difficulty and showed a good outcome of long-term follow-up. Many authors have reported SSTO with un-cemented THA [14-20]. SSTO procedure was complicated and challenging to surgeons.

In clinical experience, for Crowe IV type developmental dysplasia of the hip, we found that some cases did not need SSTO and could get reduction while others hardly could not get reduction unless SSTO. With understanding and reviewing both the Crowe and Hartofilakidis classification systems, we presumed that the presence or absence of secondary false acetabulum may result in different biomechanical patterns of proximal femur and pelvis, which may lead to differences in the anatomical morphology of the proximal femur, affecting the depth of implantation of the prosthesis, and thus affecting the reduction and necessity of SSTO.

Currently, the S-ROM prosthesis was recognized as the best prosthetic option for severe femoral deformity such as Crowe type IV DDH. This article was designed 
to investigate the surgical options between the presence and absence of secondary false acetabulum in the treatment of Crowe IV DDH with the S-ROM prosthesis. If difference presents, it is necessary to classify the Crowe IV type DDH into two subtypes depending on if false acetabulum exists or not, which facilitates and instructs surgery.

\section{Materials and methods}

This study was performed with the Investigational Review Board approval. In keeping with the requirements of a retrospective description and review, informed consent was not required. A retrospective cohort study included 32 patients with Crowe type IV DDH underwent THA (34 hip joints) from January 2011 to December 2015. The mean follow-up time was 30 months (from 24 to 70 months).

According to the presence or absence of secondary false acetabulum on the acetabulum side, from January 2011, all subjects were progressively chosen to join this program. There were five males and 27 females with an average age of $42.26 \pm 11.20$ years (22-68 years), 6 cases of unilateral Crowe type IV DDH, and 19 cases of bilateral Crowe type IV DDH. As of April 2016, all patients had completed follow-up, which included HSS score, X-ray films, and complications. Twenty-eight hips were divided into no false acetabulum group, while 16 hips into the false acetabulum group. In the no false acetabulum group, 14 females and two males had an average age of $39.85 \pm 11.46$ years old. In the false acetabulum group, 23 females and five males had an average age of $42.01 \pm 10.83$ year old. The clinical data of age and gender pencertage of the two groups were compared in Table 1 . The difference was not statistically significant.

\section{Diagnostics, inclusion, and exclusion criteria}

Diagnostic criteria are according to the Crowe classification system; Crowe type IV DDH with high dislocation was defined as the ratio of the vertical distance from the head-neck junction to the teardrop line to the diameter of the femoral head $>100 \%$ or the ratio of the vertical distance to the height of the pelvis $>20 \%$ [1]. Inclusion criteria are strictly in accordance with Crowe type IV. Exclusion criteria are high dislocation of the hip secondary to septic arthritis. The indication for THR in these patients was severe hip pain and impairment of daily

Table 1 Different groups depending on if false acetabulum exists or not

\begin{tabular}{|c|c|c|c|c|}
\hline \multirow[t]{2}{*}{ Group } & \multirow[t]{2}{*}{ No } & \multicolumn{2}{|l|}{ Gender } & \multirow{2}{*}{$\begin{array}{l}\text { Age (mean } \\
\pm S D \text {, year) }\end{array}$} \\
\hline & & Female & Male & \\
\hline False acetabulum & 16 & 14 & 2 & $42.01 \pm 10.83$ \\
\hline No false acetabulum & 28 & 23 & 5 & $39.85 \pm 11.46$ \\
\hline Statistical value & & \multicolumn{2}{|l|}{$x^{2}=0.22$} & $t=0.61$ \\
\hline$P$ value & & \multicolumn{2}{|l|}{$P>0.05$} & $P>0.05$ \\
\hline
\end{tabular}

activity, regardless of the presence of iliofemoral OA. All medical records were reviewed.

The criterion on how we could judge if false acetabulum existed was evaluated on the pelvis plain X-ray. If the femoral head exceeded or reached the lateral ilium wing margin and a white line with indication of osteosclerosis emerged under long-term pressure on plain radiograph, we believed that the femoral head articulated with a false acetabulum. If the femoral head did not reach the lateral ilium wing margin or we could not see any indicating sign of osteosclerosis on plain radiograph, we concluded that no false acetabulum and the femoral head were free floating within the gluteal musculature. See Fig. 1.

\section{Treatment}

The femoral prosthesis of both groups used a modular S-ROM stem ( Depuy, Warsaw, IN, USA), while on acetabular side, Gription Pinnacle cup ( Depuy, Warsaw, IN, USA) was used. The acetabular side was additionally fixed with a screw. The 28-mm-diameter ceramic head was used. Twenty-one hips were applied with SSTO during THA and 23 hips needed not SSTO. All procedures were performed by the same experienced joint surgeon, and the procedure used the posterolateral approach. The external rotation muscle group was separated during operation, the joint capsule was opened, and the femoral head was removed from the base of the femoral neck. The iliopsoas and part of the gluteus maximus are released from the femoral attachments, and the joint capsule was removed to expose the original acetabulum. A ceramic-to-ceramic interface with a 28 -mm-diameter head could be used by grinding a 43- or $44-\mathrm{mm}$ hole from a relatively bone stock area in the posterior and lower parts of the original acetabulum. Gription Pinnacle cup with 44-mm diameter could be used.

The proximal femur neck osteotomy was performed at the femoral neck, and the distance between the osteotomy plane and the lesser trochanter plane was determined on the dislocation height. Gradually to the distal but still above the lesser trochanter plane until the femoral stem, trial could be safely inserted. After proximal femur osteotomy, reduction was attempted. If we could get a reduction, true prosthetic components were placed. If reduction was still difficult, SSTO would be chosen (Fig. 2).

\section{SSTO}

The distal femur and proximal medullary cavity were prepared and the S-ROM trial was implanted. The distance between the center of rotation of the femoral head and the center of the acetabulum was measured under traction, and the osteotomy was performed at $10 \mathrm{~mm}$ or $20 \mathrm{~mm}$ under the small trochanter plane. The bone length that we wanted to remove was the distance between the center of rotation of the femoral head and the 

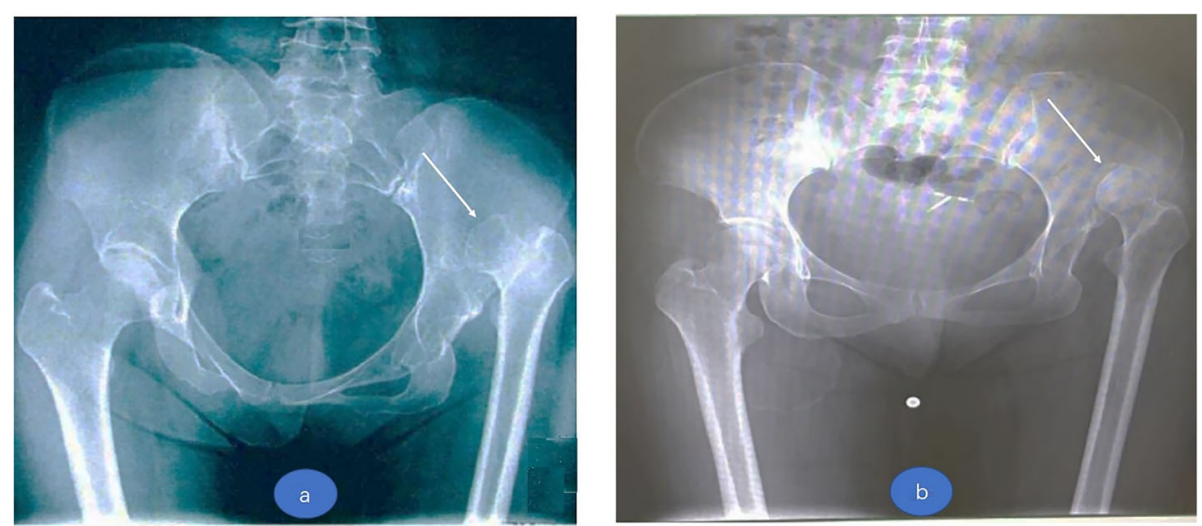

Fig. 1 a A Crowe IV hip without pseudoacetabulum and dislocated femoral head at posterior ilium place. b A Crowe IV hip with pseudoacetabulum and a white line with indication of osteosclerosis emerged at acetabulum side (white arrows point)

center of the acetabulum minus $15 \mathrm{~mm}$ (half a femoral head diameter). If a femoral split occurred during the operation, the distal and proximal ends of the femoral trochanter and the osteotomy were tied with steel wire, and the prosthetic test was inserted until the cortex is attached. After the trial implantation, the length and stability of the lower limbs were checked, and the true femoral stem was implanted.

\section{Postoperative rehabilitation}

In order to protect the nerve vascular tissue, the knees were bent $30^{\circ}$. In the morning of the day after surgery, with crutches, patients were encouraged to move down on the ground, which may be partially loaded. At 6 weeks after surgery, the patient could move with full weight. Patients were encouraged to exercise as early as possible to correct pelvic tilt and gait.

\section{Evaluation after surgery}

Patients were followed up 1 month, 3 months, 6 months, 12 months, and annually later beyond 1 year after surgery by outpatient review, including physical examination, $\mathrm{X}$ - ray, and observation of healing at the osteotomy site and the presence of the prosthesis. Before and after surgery, Harris score of the hip joint was recorded. Postoperative complications such as nerve injury, hip dislocation, and periprosthetic fractures were recorded.

\section{Statistical processing}

All the collected data were statistically processed using SPSS 21st version software (SPSS Inc., Chicago, IL, USA), and the $t$ test of quantitative data designed in groups was used to compare the Harris score between the two groups before surgery and during follow-up of patients in both groups. Preoperative and postoperative Harris scores of hip joints were compared by $t$ test of paired design quantitative data. By chi-square test, SSTO was compared between the two groups. Chi-square test was used to compare the incidence of complications in the two groups. All statistical tests were performed at $95 \%$ bilateral significance level, $P<0.05$, and the difference was statistically significant.
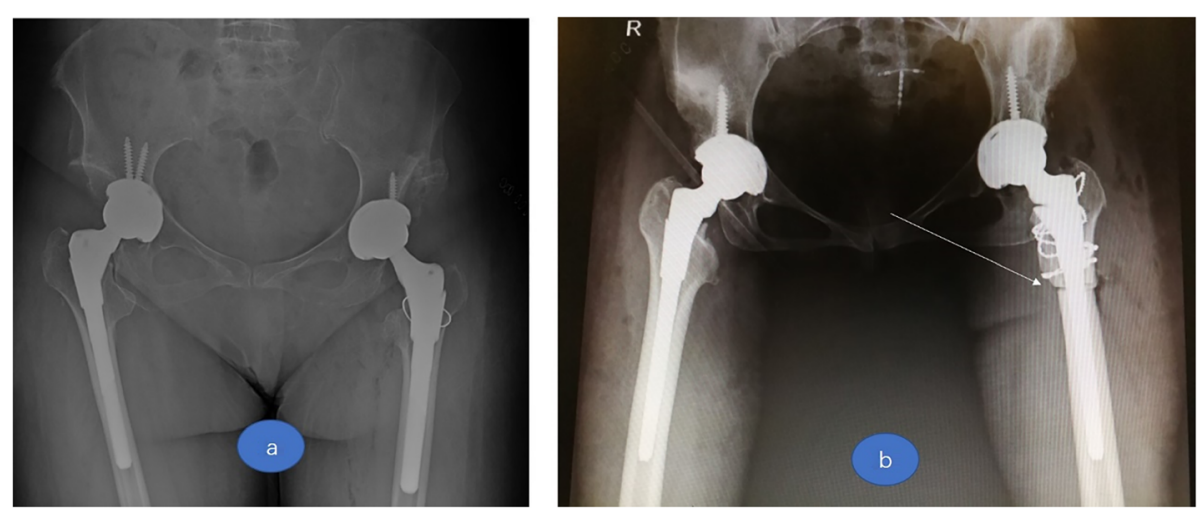

Fig. 2 a A Crowe IV hip on right side without SSTO in THA. b A Crowe IV hip on right side with SSTO in THA (white arrow points) 


\section{Results}

SSTO application group with false acetabulum of 16 hips, 3 hips used the SSTO, $18.75 \%$, while in the group without false acetabulum, 8 of the 28 hips were treated with SSTO, accounting for $28.57 \%$. The differences in SSTO used between the two groups were statistically significant (chi-square $=11.33$ ). The proportion of SSTO used in the non-secondary false acetabulum group was higher than that in the secondary acetabulum formation group. See Table 2

\section{HSS score evaluation}

HSS scores of two groups were compared, and outcomes are shown in Table 3. Preoperative and postoperative (at the last follow-up) Harris scores showed no significant difference in pain, function, or total score between the two groups. Harris scores of all patients after surgery were higher than those before surgery (Table 3 ).

\section{Discussion}

For Crowe type IV DDH, it is difficult to restore the natural rotation center at the original acetabulum place, which has been demonstrated effective and sure of good long-term outcome. The consequence that follows the restoration of the rotation center at original acetabulum place challenges surgeons to avoid nerve injury, and limbs length discrepancy. SSTO procedure is placed on the docket to solve these problems. The necessity of SSTO is determined by varied factors such as dislocation distance, presence or absence of pseudoacetabulum, and so on. Hartofilakidis [6] have previously described two subtypes of high dislocation of the hip, depending on the presence $(\mathrm{C} 1)$ or absence $(\mathrm{C} 2)$ of a false acetabulum and reported the different results, especially in survivorship, between $\mathrm{C} 1$ and $\mathrm{C} 2$ hips regardless of surgery procedure [7]. Taking advantages of the subtype content from the Hartofilakidis classification to Crowe classification since Crowe classification addressed the dislocation distance precisely in ration of proximal displacement distance of the femoral head in relation to the height of the pelvis or the femoral head, the Crowe classification is more effective and efficient as a surgery guideline with consideration of both dislocation distance and presence of pseudoacetabulum. In this study, we determined whether the SSTO application in THA differs between the two

Table 2 Comparison in SSTO application between different groups

\begin{tabular}{llll}
\hline Group & No & \multicolumn{2}{l}{ SSTO application } \\
\cline { 3 - 4 } & & SSTO & No SSTO \\
\hline False acetabulum & 16 & 13 & 3 \\
No false acetabulum & 28 & 8 & 20 \\
Statistical value & & $X^{2}=11.33$ & \\
$P$ value & & $P<0.05$ & \\
\hline
\end{tabular}

aforementioned subtypes of Crowe IV hips. Although there are no other studies in the literature comparing SSTO application in THA in high dislocation subtypes, this outcome seems reasonable, because Crowe IV hips without pseudoacetabulum have more affected femur development and eventual morphology. If pseudoacetabulum exists, the femur has a coverage with compressive stress to develop a relatively approximate normal hip morphology compared with hips without pseudoacetabulum [21]. The presence or absence of secondary acetabulum will lead to different stress patterns of the proximal femur, affecting the development of the proximal femur medullary cavity, and then lead to the difference in anatomical morphology of the proximal femur and the choice of surgical methods. This can answer why some do not need SSTO while others need SSTO in clinical work. Ma et al. [9] reported a similar outcome.

A number of classification systems $[1,4,6,22,23]$ have been published to grade osteoarthritis secondary to DDH. Crowe et al. [1] defined a four-stage system classifying the degree of dislocation in terms of the percentage of proximal displacement of the femoral head in relation to the height of the pelvis. Hartofilakidis et al. $[4,6]$ used the pathology of the dysplastic acetabulum to distinguish between three different types of dysplasia, discriminating between a primary and secondary acetabulum and the relation of the head in relation to these structures. Both systems have shown a better reproducibility.

Both Hartofilakidis and Crowe classification systems have deficiencies. The main shortcoming of this Crowe classification is that it cannot reflect the hip pathological changes and consequent biomechanical reconstruction which may have a very long and gradual process. Hartofilakidis et al proposed three different types and later divided low dislocation and high dislocation into two subtypes. The shortcoming of the Hartofilakidis classification system is that it is concerned on only the morphology and insufficient lacks of guidance for surgical treatment since its classification criteria are not precise in form of calculation. We can add these subtypes regarding on the presence or absence of pseudoacetabulum to the Crowe classification system. With combination of these two popular classification systems, it can be more effective and efficient as guidance for DDH treatment.

Several limitations should be considered. First, the study was retrospective in nature and thus was the subject to its inherent limitations and biases. Second, the results were from a single institution and the sample size was not large. However, no previous study has a large sample size because DDH has high morbidity in Asia. Third, the presence or absence of pseudoacetabulum was judged by surgeons without strict criteria, which led to bias. Further prospective studies with larger cohorts are required to validate these results. 
Table 3 Comparison on HSS score

\begin{tabular}{|c|c|c|c|c|c|c|c|}
\hline \multirow[t]{2}{*}{ Group } & \multirow[t]{2}{*}{ No } & \multicolumn{3}{|l|}{ Pre-surgery } & \multicolumn{3}{|l|}{ Post-surgery } \\
\hline & & Pain & Function & Total & Pain & Function & Total \\
\hline False & 16 & $23.85 \pm 10.73$ & $33.35 \pm 9.43$ & $57.65 \pm 15.24$ & $39.56 \pm 4.31$ & $51.23 \pm 5.26$ & $88.56 \pm 4.79$ \\
\hline No & 28 & $27.23 \pm 11.46$ & $30.26 \pm 11.24$ & $54.23 \pm 16.38$ & $41.89 \pm 3.29$ & $49.38 \pm 4.39$ & $86.43 \pm 5.01$ \\
\hline$t$ value & & 0.96 & 0.93 & 0.68 & 2.02 & 1.25 & 1.38 \\
\hline$P$ value & & $P>0.05$ & $P>0.05$ & $P>0.05$ & $P>0.05$ & $P>0.05$ & $P>0.05$ \\
\hline
\end{tabular}

\section{Conclusions}

This study reveals that pseudoacetabulum is an important factor determining SSTO application in total hip arthroplasty for Crowe IV hips. We can add these subtypes regarding on the presence or absence of pseudoacetabulum to the Crowe classification system. With combination of these two popular classification systems, it can be more effective and efficient as guidance for DDH treatment option.

\section{Abbreviations}

DDH: Developmental dysplasia of the hip; SSTO: Subtrochanteric shortening osteotomy; THA: Total hip arthroplasty

\section{Acknowledgements}

The authors would like to thank all the staff from the participating departments and clinics.

\section{Authors' contributions}

YW and HP designed the study. TW and CX collected the data. GZ, HP, and YW were involved in the manuscript writing, literature search, data interpretation, and data monitoring. HP was responsible for the data collection and analysis. All authors read and approved the final manuscript.

\section{Funding}

None. No benefits or funds were received in support of the study.

\section{Availability of data and materials}

The patients' data were collected in the Chinese PLA General Hospital. The datasets used and/or analyzed during the current study are available from the corresponding author on reasonable request.

\section{Ethics approval and consent to participate}

This study was conducted with approval from the Ethics Committee of the Chinese PLA General Hospital. Furthermore, this study was performed with the Investigational Review Board approval. In keeping with the requirements of a retrospective description and review, informed consent was not required.

\section{Consent for publication}

We have obtained consent to publish from the participants.

\section{Competing interests}

The authors declare that they have no competing interests.

Received: 2 January 2019 Accepted: 29 May 2019

Published online: 03 July 2019

\section{References}

1. Crowe JF, Mani VJ, Ranawat CS. Total hip replacement in congenital dislocation and dysplasia of the hip. J Bone Joint Surg Am. 1979;61(1): 15-23.

2. Eftekhar NS. Total hip arthroplasty. St. Louis: CV Mosby; 1993. p. 925.

3. Hartofilakidis G, Stamos K, loannidis TT. Low friction arthroplasty for old untreated congenital dislocation of the hip. J Bone Joint Surg Br. 1988;70(2): $182-6$.
4. Hartofilakidis G, Stamos K, Karachalios T, Ioannidis TT, Zacharakis N. Congenital hip disease in adults. Classification of acetabular deficiencies and operative treatment with acetabuloplasty combined with total hip arthroplasty. J Bone Joint Surg Am. 1996;78(5):683-92.

5. Kerboul M, Mathieu M, Sauzieres P. Total hip replacement for congenital dislocation of the hip. In: Postel M, Kerboul M, Evrard J, Courpied JP, editors. Total hip replacement. New York: Springer; 1987.

6. Eftekhar N. Principles of total hip arthroplasty. St. Louis: C V Mosby; 1978. p. 437-55.

7. Hartofilakidis G, Yiannakopoulos CK, Babis GC. The morphologic variations of low and high hip dislocation. Clin Orthop Relat Res. 2008;466(4):820-4. https://doi.org/10.1007/s11999-008-0131-9.

8. Hartofilakidis G, Babis GC, Lampropoulou-Adamidou K, Vlamis J. Results of total hip arthroplasty differ in subtypes of high dislocation. Clin Orthop Relat Res. 2013;471(9):2972-9. https://doi.org/10.1007/s11999013-2983-x.

9. Brunner A, Ulmar B, Reichel H, Decking R. The Eftekhar and Kerboul classifications in assessment of developmental dysplasia of the hip in adult patients. Measurement of inter- and intraobserver reliability. HSS J. 2008;4(1): 25-31. https://doi.org/10.1007/s11420-007-9066-z.

10. Ma HY, Zhou YG, Zheng C, Cao WZ, Wang S, Wu WM, Du YQ. New classification of Crowe type IV developmental dysplasia of the hip. Zhongguo Gu Shang. 2016;29(2):119-24.

11. lida H, Matsusue $Y$, Kawanabe K, Okumura H, Yamamuro T, Nakamura T. Cemented total hip arthroplasty with acetab $\neg$ ular bone graft for developmental dysplasia. Long-term results and survivorship analysis. J B Jt Surg Br. 2000;82:176-84

12. Klisic P, Jankovic L. Combined procedure of open reduc $\neg$ tion and shortening of the femur in treatment of congenital dislocation of the hips in older children. Clin Orthop Rel Res. 1976;119:60-9.

13. Sponseller PD, McBeath AA. Subtrochanteric osteotomy with intramedullary fixation for arthroplasty of the dysplastic hip. A case report. J Arthropl. 1988; 3:351-4.

14. Chareancholvanich K, Becker DA, Gustilo RB. Treatment of congenital dislocated hip by arthroplasty with femoral shorten $\neg$ ing. Clin Orthop Rel Res. 1999;360:127-35.

15. Kerboull M, Hamadouche M, Kerboull L. Total hip arthro $\neg$ plasty for Crowe type IV developmental hip dysplasia: a long-term follow-up study. J Arthropl. 2001;16:170-6.

16. Masonis JL, Patel JV, Miu A, Bourne RB, McCalden R, Macdonald SJ, Rorabeck $\mathrm{CH}$. Subtrochanteric shortening and de-rotational osteotomy in primary total hip arthroplasty for patients with severe hip dysplasia: 5-year followup. J Arthropl. 2003;18:68-73.

17. Reikeraas O, Lereim P, Gabor I, Gunderson R, Bjerkreim I. Femoral shortening in total arthroplasty for completely dislocated hips: 3-7 year results in 25 cases. Acta Orthop Scand. 1996:67:33-6.

18. Howie CR, Ohly NE, Miller B. Cemented total hip arthro $\neg$ plasty with subtrochanteric osteotomy in dysplastic hips. Clin Orthop Rel Res. 2010;468: 3240-7

19. Kawai T, Tanaka C, Ikenaga M, Kanoe H. Cemented total hip arthroplasty with transverse subtrochanteric shorten-ing osteotomy for Crowe group IV dislocated hip. J Arthropl. 2011;26:229-35.

20. Charity JA, Tsiridis E, Sheeraz A, Howell JR, Hubble MJ, Timperley AJ, Gie GA. Treatment of Crowe IV high hip dysplasia with total hip replacement using the Exeter stem and shortening de-rotational subtrochanteric osteotomy. J B Jt Surg Br. 2011:93:34-8.

21. Xu H, Zhou Y, Liu Q, Tang Q, Yin J. Femoral morphologic differences in subtypes of high developmental dislocation of the hip. Clin Orthop Relat Res. 2010;468(12):3371-6. https://doi.org/10.1007/s11999-010-1386-5. 
22. Kerboul M, Mathieu M, Sauzieres P. Total hip replacement for congenital dislocation of the hip. In: Postel M, Kerboul M, Evrard J, Courpied JP, editors. Total hip replacement. Berlin Heidelberg New York: Springer; 1987. p. 51-66.

23. Mendes DG, Said MS, Aslan K. Classification of adult congenital hip dysplasia for total hip arthroplasty. Orthopedics. 1996;19:881-7.

\section{Publisher's Note}

Springer Nature remains neutral with regard to jurisdictional claims in published maps and institutional affiliations.

Ready to submit your research? Choose BMC and benefit from:

- fast, convenient online submission

- thorough peer review by experienced researchers in your field

- rapid publication on acceptance

- support for research data, including large and complex data types

- gold Open Access which fosters wider collaboration and increased citations

- maximum visibility for your research: over $100 \mathrm{M}$ website views per year

At $B M C$, research is always in progress.

Learn more biomedcentral.com/submissions 